\title{
Amélioration du transfert thermique par optimisation de la géométrie d'une conduite de révolution
}

\author{
Rachid Sehaqui ${ }^{a}$, Meryem Sijelmassi et JaÂfar Khalid Naciri \\ Faculté des Sciences Ain Chock, UFR de mécanique BP 5366, Maarif, Casablanca, Maroc
}

Reçu le 6 novembre 2003, accepté le 9 janvier 2004

\begin{abstract}
Résumé - L'objectif de ce travail est de déterminer une forme de profil de vitesse à l'entrée d'un tube circulaire, ainsi que la forme géométrique de la paroi de la conduite correspondant, permettant de maximiser le transfert de chaleur à l'intérieur de la conduite. On considère la convection mixte, bidimensionnelle, laminaire pour un fluide newtonien, en géométrie axisymetrique. Le profil de vitesse axiale à l'entrée du tube optimum, est obtenu numériquement, en résolvant le système d'équations aux dérivées partielles en coordonnées cylindriques, par une méthode aux différences finies. Pratiquement ce profil est obtenu en modifiant sur une longueur donnée, la forme de la paroi de la conduite créée par des perturbations particulières du profil amont, ce qui permet de générer le profil choisi.
\end{abstract}

Mots clés : Conduite déformée / perturbations / profil de vitesse / transfert thermique

\begin{abstract}
Increase heat transfer by optimization revolution geometry. The objective of this work is to study the influence of the velocity profiles and geometrical on the heat transfer. We consider a two-dimensional mixed convection in a circular tube heated from below for a Newtonian fluid in the laminar regime. The optimum velocity profile is obtained numerically. A semi-analytical method is used to obtain the geometrical modification of the duct walls.
\end{abstract}

Key words: Heat transfer / perturbations / velocity profile / channel flows

\section{Introduction}

Le transfert thermique par convection mixte à l'intérieur d'un tube circulaire, présente un intérêt considérable, en vue d'applications technologiques telles que : dépôt chimique des couches minces, refroidissement des pièces électroniques [1-7], augmentation de l'efficacité d'un échangeur de chaleur, calcul de l'énergie d'un capteur solaire et plusieurs autres domaines des sciences de l'ingénieur. Les phénomènes qui apparaissent dans le cas de la convection mixte en géométrie axisymetrique sont complexes et dépendent en plus de la géométrie de la conduite, de plusieurs paramètres : le nombre de Reynolds $R e$, le nombre de Rayleigh $R a$, et le nombre de Prandtl $\mathrm{Pr}$. Les conditions aux limites, tant celles fixant le profil de vitesse à l'entrée du tube, que celles relatives à la géométrie de la conduite, ont une influence sur le transfert thermique. Si de nombreux travaux ont cherché

\footnotetext{
a Auteur correspondant : sehaqui@hotmail.com
}

à corréler le transfert thermique aux conditions aux limites thermiques imposées aux parois du tube [8-10], ou à la forme géométrique de la conduite [11-13], peu ont concerné l'influence des conditions amont et aval sur ce même transfert. L'objectif de cette étude est de déterminer numériquement une forme de profil de vitesse axiale imposée à l'entrée du tube permettant de maximiser le transfert thermique. Cette forme de profil est obtenue par une déformation géométrique de la conduite sur une longueur donnée. Dans ce but, nous avons à partir d'une résolution numérique des équations complètes de mouvement, déterminé une forme de profil de vitesse à l'entrée de la conduite, permettant de rendre maximum le nombre de Nusselt. Une méthode semi-analytique détaillée dans [14] est utilisée dans ce travail. Elle permet de générer à travers des modifications de géométrie, un profil de vitesse arbitraire préalablement défini dans une section donnée de la conduite. Cette forme de profil correspond à une modification sur une longueur donnée, de la forme géométrique de la paroi de la conduite. 


\section{Recherche du profil optimum : résolution numérique}

\subsection{Formulation mathématique du problème}

Dans le système de coordonnées cylindriques $(\tilde{r}, \tilde{\theta}, \tilde{z})$ on considère l'écoulement axisymétrique plan d'un fluide newtonien incompressible de viscosité cinématique $\nu$, dans un tube circulaire de très grande longueur $L_{0}$ et de rayon $R_{0}$. Les conditions aux limites thermiques imposées sont : une température $T_{\mathrm{c}}$ pour $r^{*}=R_{0}$ et un flux de chaleur nul sur l'axe. On introduit les quantités adimensionnelles suivantes :

$$
\begin{aligned}
& t=\varepsilon \frac{t^{*} U_{0}}{R}, \quad r=\frac{r^{*}}{R_{0}}, \quad z=\varepsilon \frac{z^{*}}{R}, \\
& V_{r}=\frac{V_{r}^{*}}{\varepsilon U_{0}}, \quad V_{z}=\frac{V_{z}^{*}}{U_{0}}, \quad p=\frac{\varepsilon R_{0} p^{*}}{\nu \rho U_{0}}, \\
& T=\frac{T^{*}-T_{0}}{\Delta T}, \quad \omega=\frac{\omega^{*} R_{0}}{U_{0}}, \quad \psi=\frac{\psi^{*}}{R_{0}^{2} U_{0}}
\end{aligned}
$$

où $t, V_{r}, V_{z}, p, T, \omega, \psi$ désignent respectivement le temps, la vitesse radiale, la vitesse axiale, la pression, le champ de température, la fonction vorticité et la fonction de courant adimensionnelles; $\Delta T, U_{0}, \varepsilon, \rho, \nu$ représentent une différence de température de référence, la vitesse moyenne, le rapport de forme $\frac{R_{0}}{L_{0}}$, la masse volumique et la viscosité cinématique. Les équations de Navier-Stokes adimensionnelles s'écrivent sous la forme :

$$
\begin{aligned}
& \frac{\partial V_{r}}{\partial r}+\frac{V_{r}}{r}+\frac{\partial V_{z}}{\partial z}=0 \\
& \frac{\partial V_{r}}{\partial t}+V_{r} \frac{\partial V_{r}}{\partial r}+V_{z} \frac{\partial V_{r}}{\partial z}= \\
& -\frac{\partial p}{\partial r}+\frac{1}{R e}\left(\frac{\partial^{2} V_{r}}{\partial r^{2}}+\frac{1}{r} \frac{\partial V_{r}}{\partial r}+\frac{\partial^{2} V_{r}}{\partial z^{2}}\right)+\frac{R a}{P r R e^{2}} T \\
& \frac{\partial V_{z}}{\partial t}+V_{r} \frac{\partial V_{z}}{\partial r}+V_{z} \frac{\partial V_{z}}{\partial z}= \\
& \quad-\frac{\partial p}{\partial z}+\frac{1}{R e}\left(\frac{\partial^{2} V_{z}}{\partial r^{2}}+\frac{1}{r} \frac{\partial V_{z}}{\partial r}+\frac{\partial^{2} V_{z}}{\partial z^{2}}\right) \\
& \frac{\partial T}{\partial t}+V_{r} \frac{\partial T}{\partial r}+V_{z} \frac{\partial T}{\partial z}=\frac{1}{P e}\left(\frac{\partial^{2} T}{\partial r^{2}}+\frac{1}{r} \frac{\partial T}{\partial r}+\frac{\partial^{2} T}{\partial z^{2}}\right)
\end{aligned}
$$

où $R e=\frac{U_{0} R_{0}}{\nu}$ est le nombre de Reynolds, $R a=\frac{\beta \Delta T R_{0}^{3}}{\nu \alpha}$ le nombre de Rayleigh, $\operatorname{Pr}=\frac{\nu}{\alpha}$ le nombre de Prandtl, $\alpha$ la diffusivité thermique, $g$ l'accélération de la pesanteur, $\beta$ le coefficient d'expansion thermique et $P e=R e P r$ le nombre de Peclet.

Aux équations (1)-(4) on associe les conditions aux limites dynamiques et thermiques suivantes :

$$
\begin{aligned}
V_{r}(r, z=0) & =T(r, z=0)=V_{r}(r=1, z) \\
& =V_{z}(r=1, z)=0, T(r=1, z)=1
\end{aligned}
$$

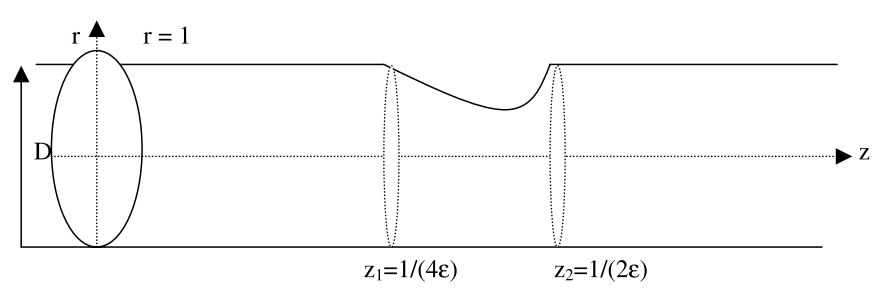

Fig. 1. Configuration géométrique étudiée et système de coordonnées.

$$
\begin{aligned}
& \frac{\partial V_{r}}{\partial z}=\frac{\partial^{2} V_{z}}{\partial z^{2}}=\frac{\partial T}{\partial z}=0 \quad \text { pour } \quad z=\frac{L_{0}}{R_{0}} \text { et } 0 \leq r \leq 1, \\
& V_{r}=V_{z}=\frac{\partial T}{\partial r}=0 \quad \text { pour } \quad 0 \leq z \leq \frac{L_{0}}{R_{0}} \text { et } r=0
\end{aligned}
$$

Le système formé des équations (1)-(4) et des conditions aux limites (5), est résolu numériquement en formulation vorticité fonction de courant $(\omega, \psi)$.

Dans le but de mettre en évidence l'effet de la forme du profil de vitesse axiale imposé à l'entrée du tube nous considérons que la condition à l'entrée de la conduite est de la forme :

$$
V_{z}(r, 0)=V_{\mathrm{p}}(r)+v(r, 0)
$$

où $V_{\mathrm{p}}(r, 0)$ est un profil parabolique défini par :

$$
V_{\mathrm{p}}(r)=2\left(1-r^{2}\right)
$$

$v(r, 0)$ est une fonction a priori arbitraire de $r$, qui vérifie les conditions d'adhérence aux parois, de débit nul et d'axisymétrie de sorte que :

$$
\begin{aligned}
v(r=1, z) & =0 \\
\left(\frac{\partial v(r, 0)}{\partial r}\right)_{r=0} & =0 \\
\int_{0}^{1} r v(r, 0) \mathrm{d} r & =0
\end{aligned}
$$

\subsection{Solution numérique}

Le système formé des équations (1)-(4) et des conditions aux limites (5) est discrétisé suivant un schéma aux différences finies utilisant un maillage uniforme dans le domaine de calcul. Les dérivées spatiales des équations de transport pour la vorticité $\omega$ et l'énergie $T$ sont approximées par un schéma centré du second ordre. Les équations (2)-(4) sont paraboliques par rapport au temps et elliptiques par rapport aux coordonnées spatiales. Elles ont été intégrées par la méthode ADI (Alternating Directions Implicit). La fonction de courant $\psi$ est déterminée par application de la méthode SOR (Successive Over Relaxation). Dans le cadre de cette étude on cherche à 
déterminer un profil de vitesse à l'entrée du tube permettant de rendre maximum le nombre de Nusselt global. Pour cela on superpose au profil de vitesse axial à l'entrée du tube une perturbation qui peut être exprimée à l'aide d'un développement en séries de Fourier, en fonction des amplitudes des perturbations symétriques $b_{k}$ et antisymétriques $a_{k}$ sous la forme :

$$
v(r, z)=\sum_{k=1}^{k=N}\left[a_{k} \sin (2 k \pi r)+b_{k} \cos (2 k \pi r)\right] \mathrm{e}^{-\lambda_{k} z}
$$

où les $\lambda_{k}$ sont des valeurs propres définies par :

$$
\lambda_{k}=\frac{4(k \pi)^{2}}{R e} \quad(k=1,2, \ldots N) .
$$

L'intensité d'échange de chaleur entre une paroi solide et un fluide en mouvement est caractérisée par le nombre de Nusselt. Le nombre de Nusselt local est défini par :

$$
\begin{aligned}
& N u(z)=\frac{-\left(\frac{\partial T}{\partial r}\right)_{r=1}}{1-T_{m}} \int_{0}^{1} V_{z}(r, z) T(r, z) \mathrm{d} r \\
& \text { avec } T_{m}(z)=\frac{\int_{0}^{1} V_{z}(r, z) \mathrm{d} r}{}
\end{aligned}
$$

L'échange de chaleur global est caractérisé par le nombre de Nusselt global :

$$
N u_{g}=\frac{R_{0}}{L_{0}} \int_{0}^{\frac{L_{0}}{R_{0}}} N u(z) \mathrm{d} z
$$

Les perturbations dynamiques superposées au profil d'entrée parabolique induisent des variations dans la forme des profils de vitesse dans toute la zone où le régime est établit. Notons que cette variation de distribution de vitesse modifie le nombre de Nusselt global (Éq. (11)) caractéristique du bilan de transfert thermique dans la conduite. Le profil de vitesse optimum est celui qui permet de maximiser le nombre de Nusselt global. L'effet de la perturbation du profil de vitesse axiale à l'entrée du tube est illustré sur la figure 2. En effet en considérant un développement de la relation (9) à l'ordre 6 , Les calculs numériques montrent pour $0 \leq a_{k} \leq 0,25$ et $0 \leq b_{k} \leq 0,25$ $(k=1,6)$ qu'il existe un extremum du nombre de Nusselt. Sur la figure 2 on remarque que les courbes correspondant à un profil de vitesse perturbé sont au-dessus de celles obtenues dans le cas où le profil est parabolique.

Sur la figure 3 représentant la variation du nombre de Nusselt global en fonction des coefficients de perturbation, on constate l'existence d'un nombre Nusselt global optimum. Le nombre de Nusselt global optimum pour un développement à l'ordre 4 (Fig. 3) est identique à celui obtenu pour un développement à l'ordre 6 (figures en annexe $3 \mathrm{~b}-3 \mathrm{c}$ ). C'est la raison pour laquelle on peut se limiter à un développement d'ordre 4 pour ce problème.

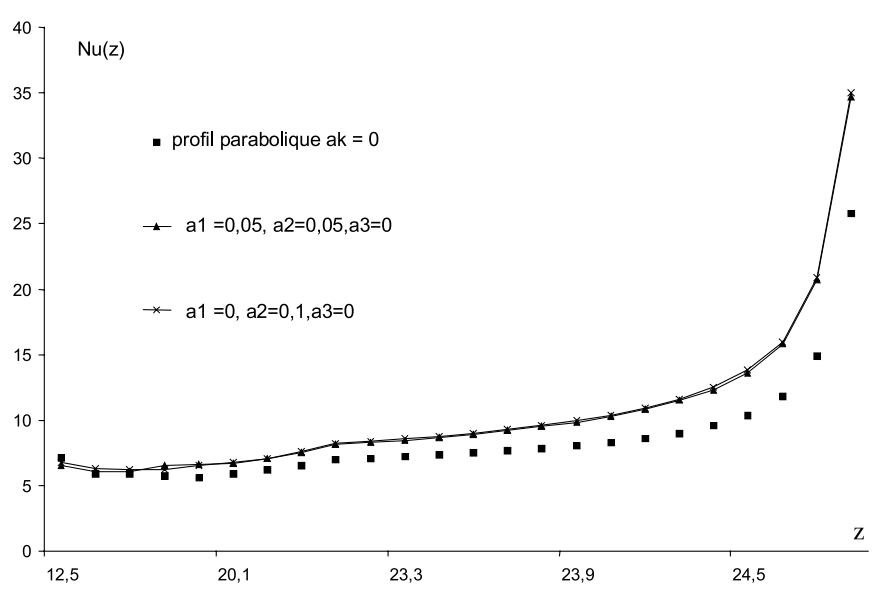

Fig. 2. Variation du nombre de Nusselt local : pour un profil de vitesse d'entrée parabolique et un profil de vitesse d'entrée déformé. $a_{4}=-a_{1}, a_{5}=-a_{2}, a_{6}=-a_{3}, R a=10^{4}, R e=600$, $\varepsilon=0,02$.

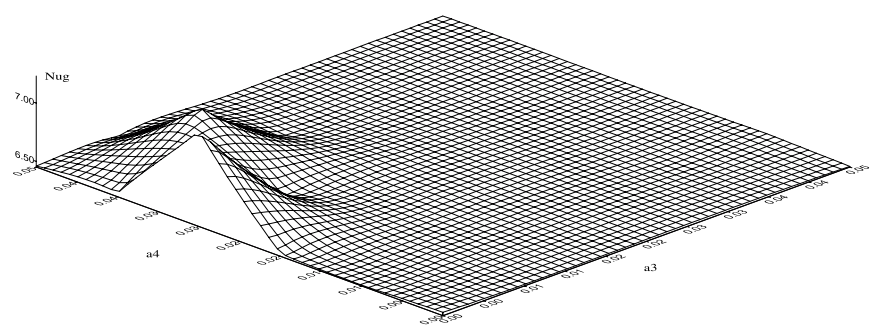

Fig. 3. Variation du nombre de Nusselt global $N u g\left(a_{3}, a_{4}\right)$, $b_{1}=b_{2}=0, a_{1}, a_{2}, a_{3}$ et $a_{4}$ vérifiant les relations issues des conditions (8), $R a=10^{4}, R e=600, \varepsilon=0,02$.

\subsection{Solution semi-analytique : détermination de la forme géométrique de paroi}

On propose à partir de l'étude des équations aux perturbations pour un écoulement stationnaire d'un fluide newtonien dans une conduite rigide à parois déformées, une méthode permettant de créer dans une section donnée de la conduite, un profil de vitesse arbitraire préalablement défini. Ce profil est obtenu en modifiant sur une longueur $L$ donnée la forme de la paroi de la conduite de sorte à créer des perturbations du profil mont permettant de générer le profil choisi. Cette méthode peut contribuer entre autres, au contrôle d'écoulement en vue de leur stabilisation ou encore à l'optimisation de formes. Dans le système des coordonnées cylindriques $(r, \theta, z)$ on considère l'écoulement axisymétrique d'un fluide newtonien incompressible dans une conduite cylindrique, rigide et déformée, d'équation $R=R(z)$ et d'axe $(O z)$. En considérant les variables adimensionnelles définies cidessus, les équations de continuité et de quantité de mouvement deviennent :

$$
\frac{\partial V_{r}}{\partial r}+\frac{V_{r}}{r}+\frac{\partial V_{z}}{\partial z}=0
$$




$$
\begin{gathered}
\varepsilon^{2} R e\left[\frac{\partial V_{r}}{\partial t}+V_{r} \frac{\partial V_{r}}{\partial r}+V_{z} \frac{\partial V_{r}}{\partial z}\right]= \\
-\frac{\partial p}{\partial r}+\left(\frac{\partial^{2} V_{r}}{\partial r^{2}}+\frac{1}{r} \frac{\partial V_{r}}{\partial r}+\varepsilon^{2} \frac{\partial^{2} V_{r}}{\partial z^{2}}-\frac{V_{r}}{r^{2}}\right) \\
\operatorname{Re}\left[\frac{\partial V_{z}}{\partial t}+V_{r} \frac{\partial V_{z}}{\partial r}+V_{z} \frac{\partial V_{z}}{\partial z}\right]= \\
-\frac{\partial p}{\partial z}+\left(\frac{\partial^{2} V_{z}}{\partial r^{2}}+\frac{1}{r} \frac{\partial V_{z}}{\partial r}+\varepsilon^{2} \frac{\partial^{2} V_{z}}{\partial z^{2}}\right)
\end{gathered}
$$

Dans le cas où $\varepsilon \ll 1$ l'écoulement est dit unidimensionnel et les équations de Navier-Stokes s'écrivent en l'absence de forces volumiques sous la forme adimensionnelle suivante :

$$
\left\{\begin{array}{l}
\frac{\partial p}{\partial r}=0 \\
R_{\mathrm{e}}\left(V_{r} \frac{\partial V_{z}}{\partial r}+V_{z} \frac{\partial V_{z}}{\partial z}\right)=-\frac{\partial p}{\partial z}+\frac{\partial^{2} V_{z}}{\partial r^{2}}+\frac{1}{r} \frac{\partial V_{z}}{\partial r} \\
\frac{\partial V_{r}}{\partial r}+\frac{V_{r}}{r}+\frac{\partial V_{z}}{\partial z}=0
\end{array}\right.
$$

Les conditions aux limites sont celles traduisant l'adhérence du fluide à la paroi, l'axisymétrie et la conservation de débit. Afin d'isoler dans le système d'équations (I) la contribution des termes correspondants à un profil de vitesse axiale de type parabolique, on introduit les transformations suivantes:

$\eta=\frac{r}{R(z)}, \quad U=V_{z} R^{2}(z), \quad V=R(z) V_{r}-\eta R R^{\prime}(z) V_{z}$,

$$
\pi=R^{4}(z) \frac{\partial p}{\partial z}
$$

On pose alors :

$$
V=V_{\mathrm{p}}+v, \quad U=u, \quad \pi=\pi_{\mathrm{p}}+p
$$

où $V_{\mathrm{p}}=2\left(1-\eta^{2}\right)$ et $\pi_{\mathrm{p}}=-8$ sont le profil de vitesse et le gradient de pression pour un écoulement à profil de vitesse axiale parabolique (dit de « Poiseuille»). Dans le cas où $u, v$ et $p$ sont suffisamment faibles pour être considérés comme des perturbations par rapport à un écoulement à profil de vitesse parabolique, les équations aux perturbations peuvent être linéarisées. Elles s'écrivent alors sous la forme :

$$
(\mathrm{II})\left\{\begin{array}{l}
\frac{\partial p}{\partial \eta}=0 \\
2 R_{\mathrm{e}}\left(1-\eta^{2}\right) \frac{\partial^{2} u}{\partial \eta \partial z}-\frac{\partial}{\partial \eta}\left[\frac{1}{\eta} \frac{\partial}{\partial \eta}\left(\eta \frac{\partial u}{\partial \eta}\right)\right]= \\
\\
R_{\mathrm{e}} \frac{32 R^{\prime}(z)}{R(z)} \eta\left(\eta^{2}-1\right)
\end{array}\right.
$$

où $R^{\prime}(z)=\frac{\mathrm{d} R(z)}{\mathrm{d} z}$. Notons que l'équation (17) du système (II) a été obtenue après dérivation par rapport à $\eta$ supprimant ainsi le terme en pression de cette relation. Les conditions aux limites adimensionnelles sont respectivement :

$$
u(1, z)=v(1, z)=0, \quad \frac{\partial u}{\partial \eta}(0, z)=0, \quad \int_{0}^{1} \eta u(\eta, z) \mathrm{d} \eta=0
$$

L'équation (17) montre qu'à chaque forme de paroi $R_{k}(z)$ correspond dans une section $z_{\mathrm{s}}$ un profil de vitesse $u_{k}\left(\eta, z_{\mathrm{s}}\right)$. La linéarité de l'équation (17) permet d'établir qu'à un profil :

$$
u\left(\eta, z_{\mathrm{s}}\right)=\sum_{k=1}^{N} \alpha_{k} u_{k}\left(\eta, z_{\mathrm{s}}\right)
$$

correspond une paroi $R(z)$ définie par :

$$
\frac{R^{\prime}(z)}{R(z)}=\sum_{k=1}^{N} \alpha_{k} \frac{R_{k}^{\prime}(z)}{R_{k}(z)}
$$

On en déduit par intégration de l'équation (19) que la paroi créant le profil $u\left(\eta, z_{\mathrm{s}}\right)$ à la section $z_{\mathrm{s}}$ est donnée par l'expression :

$$
R(z)=\prod_{k=1}^{N} R_{k}^{\alpha_{k}}(z)
$$

Cette propriété inhérente à la nature de l'équation (17) peut être exploitée en vue de répondre à la question suivante : «quelle forme de paroi $R^{*}(z)$ faut-il se donner pour créer dans une section $z^{*}$ de la conduite, un profil de vitesse ayant une forme $f\left(\eta, z^{*}\right)$ donnée? ».

La méthode proposée consiste à générer un ensemble de $N$ profils de vitesse indépendants à partir d'une succession de forme de paroi. Ces profils sont utilisés pour approximer à partir de la méthode de Gramm-Schmit le profil recherché. On établit alors la relation :

$$
f\left(\eta, z^{*}\right)=\sum_{k=1}^{N} \alpha_{k} u_{k}\left(\eta, z^{*}\right)
$$

d'où l'on déduit la forme de paroi :

$$
R^{*}(z)=\prod_{k=1}^{N} R_{k}^{\alpha_{k}}(z)
$$

\section{Contrôle du profil de vitesse par déformation de paroi}

En se donnant un profil de vitesse parabolique à l'entrée de la conduite et un profil cible (profil de vitesse optimum) à atteindre dans une section donnée, 


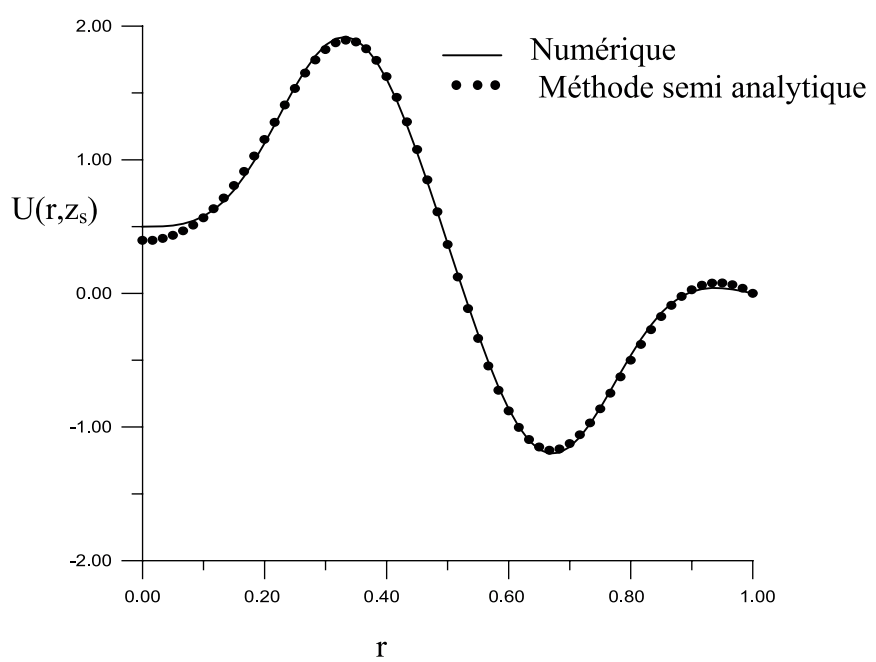

Fig. 4. Profils de vitesse.

on détermine la forme de la conduite à l'aide de la relation suivante :

$$
R\left(z^{*}\right)=\prod_{k=1}^{N} R_{k}^{\alpha_{k}}\left(z^{*}\right)
$$

Les coefficients $\alpha_{k}$ sont connus. Les formes de parois $R_{N}\left(z^{*}\right)$ sont exprimées par :

$$
R_{N}\left(z^{*}\right)=1+0,2 \sin \left(\frac{2 \pi(N-1)\left(z^{*}-z_{1}^{*}\right)}{z_{3}^{*}}\right)
$$

où $N$ est un entier, $z_{1}^{*}$, $z_{2}^{*}$ et $z_{3}^{*}=z_{2}^{*}-z_{1}^{*}$, sont des constantes définissant la zone de déformation de paroi. Après résolution et en se limitant à $N=6$, les coefficients $\alpha_{k}$ obtenus sont :

$$
\begin{aligned}
& \alpha_{1}=-885,3, \quad \alpha_{2}=27755,5, \quad \alpha_{3}=-266367,6, \\
& \alpha_{4}=853873,7, \quad \alpha_{5}=-1061148,9, \quad \alpha_{6}=446833,6
\end{aligned}
$$

La comparaison de la forme du profil de vitesse optimum avec celle obtenue par la méthode semianalytique, est illustrée sur la figure 4. La courbe en traits pleins représente le profil de vitesse optimum obtenu numériquement, alors que les symboles ronds représentent le profil de vitesse obtenu à partir de la relation (21), donnant la forme de paroi $R\left(z^{*}\right)$. Les résultats obtenus sont en accord remarquables avec le profil exacte.

\section{Conclusions}

Dans ce travail grâce à une résolution numérique des équations de la convection mixte dans une conduite rigide cylindrique, nous avons :

1. Montré l'influence de la perturbation du profil de vitesse axiale à l'entrée de la conduite sur le coefficient de transfert thermique caractérisé par le nombre de Nusselt global.
2. Déterminé des coefficients de perturbation permettant de maximiser le nombre de Nusselt global.

3. Déterminé par une méthode semi-analytique la forme géométrique de paroi permettant de générer le profil de vitesse optimum, obtenu numériquement.

Ce résultat peut s'appliquer à la résolution de quelques problèmes d'optimisation, intéressant le domaine des sciences de l'ingénieur et la stabilité des écoulements en conduite et en canal rectangulaire.

\section{Références}

[1] A. Zebib, Y.K. Wo, A two-dimensional conjugate heat transfer model for forced air cooling of an electronic device, Transactions of ASME J. Electronic Packaging 111 (1989) 41-45

[2] K. Hijikata, W. Nakayama, T. Nagasaki, R. Kurazume, K. Fushinobu, A study on heat transfer from small heating element in an integrated circuit chip, Proc. of the 3rd ASME/JSME Thermal Engineering Joint Conf. 4, 1991, pp. $93-98$

[3] T. Nagasaki, K. Hijikata, K. Fishinobu, P.E. Phelan, Numerical simulation of the conjugate direct cooling of a micro heat generating element, Proc. ASME/JSME Conf. on Electronic Packaging 1, 1992, pp. 217-223

[4] K. Fushinobu, P.E. Phelan, T. Nagasaki, K. Hijikata, M.I. Flik, Periodic steady-state thermal analysis of a high-Tc superconducting microbolometer, Trans. ASME J. Heat Transfer. 116 (1994) 278-275

[5] K. Fushinobu, K. Hijikata, Y. Kurosaki, Heat transfer regime map for electronic devices cooling, Int. J. Heat Mass Transfer. 39 (1996) 3139-3145

[6] B.A. Jubran, S.A. Swiety, M.A. Hamdan., Convective heat transfer and pressure drop characteristics of various array configurations to simulate the cooling of electronic modules, Int. J. Heat Mass Transfer. 39 (1996) 3519-3529

[7] M. Hasnaoui, E. Bilgen, P. Vasseur, Natural convection above an array open cavities heated from below, Numerical Heat Transfer. Part A 18 (1991) 463-482

[8] J.N.N. Quaresma, R.M. Cotta, Exact solutions for thermally developing tube flow with variable wall heat flux, Int. Comm. Heat Mass Transfer. 41 (1994) 729-742

[9] Dae-Young Lee, Sang-Jin. Park, Sung. Tack. Ro, Heat transfer by oscillating flow in circular pipe with a sinusoidal wall temperature distribution, Int. J. Heat Mass Transfer. 38 (1995) 2529-2537

[10] P. Stefano, An analytical approach to fully developedheating of laminar flows in circular pipes, Int. Comm. Heat Mass Transfer. 22 (1995) 815-824

[11] T. Nishimura, N. Kojima, Mass transfer enhancement in symmetric sinusoidal wavy-walled channel for pulsatile flow, Int. J. Heat Mass Transfer. 38 (1995) 1719-1731

[12] G. Russ, H. Beer, Heat transfer and flow field in a pipe with sinusoidal wavy surface-I numerical investigation, Int. J. Heat. Mass Transfer. 40 (1997) 1061-1070

[13] G. Russ, H. Beer, Heat transfer and flow field in a pipe with sinusoidal wavy surface-II Experimental investigation, Int. J. Heat. Mass Transfer. 40 (1997) 1071-1081

[14] M. Sijelmassi, J. Khalid Naciri, Contrôle des profils de vitesse par déformation de parois dans une conduite, C. R. Acad. Sci. Paris série IIb 330 (2002) 153-158 